\title{
Error Bounds for Eigenvectors of Self-Adjoint Operators*
}

\author{
Norman W. Bazley and David W. Fox**
}

(November 8, 1961)

\begin{abstract}
Error estimates are given for $\left\|u_{i}-\varphi\right\|$, where $u_{i}$ is a normalized eigenvector of a selfadjoint operator $A$ and $\varphi$ is a normalized approximating vector. The estimates contain upper and lower bounds to certain of the eigenvalues of $A$ together with the terms $(A \varphi, \varphi)$ and $\left(A \varphi, A_{\varphi}\right)$.
\end{abstract}

\section{Introduction}

In this paper we give some estimates for the error in norm between eigenvectors of a self-adjoint operator and approximating vectors. Our estimates involve upper and lower bounds to certain of the eigenvalues together with terms in the approximating vector itself. Estimates using upper and lower bounds alone have been given by Löwdin and Shull ${ }^{1}$ and Weinberger. ${ }^{2}$ Other estimates involving the same quantities used here have been given by Kato, ${ }^{3}$ Kryloff, ${ }^{4}$ Trefftz, ${ }^{5}$ and Weinberger. ${ }^{6}$ Such error bounds are useful in the estimation of quantum mechanical expectation values, error estimates for approximate solutions of $A u=f$, and other applications.

\section{Simple Eigenvalues}

We suppose $A$ to be a self-adjoint operator with domain $D_{A}$ in a separable Hilbert space $\mathfrak{S}$ having inner product $(u, v)$. $A$ is assumed to be bounded below and to have at least the initial part of its spectrum consisting of eigenvalues of finite multiplicity. These eigenvalues are considered to be ordered in a nondecreasing sequence, $\lambda_{1} \leq \lambda_{2} \leq \lambda_{3} \leq$. . ., in which each eigenvalue appears according to its multiplicity. We denote the corresponding orthonormal eigenvectors by $u_{1}, u_{2}, u_{3}, \ldots$.

We wish to estimate the difference in norm between an eigenfunction $u_{i}$ corresponding to a nondegenerate eigenvalue $\lambda_{i}$ and a given vector $\varphi$ in $\mathfrak{D}_{A}$. For convenience we will consider $\varphi$ to be normalized but undetermined to the extent of a scalar multiplier of magnitude one. The norm difference between $u_{i}$ and $\varphi$ is given by

$$
\left\|\varphi-u_{i}\right\|=\left(\varphi-u_{i}, \varphi-u_{i}\right)^{\frac{1}{2}}=\sqrt{ } 2\left[1-\operatorname{Re}\left(\varphi, u_{i}\right)\right]^{\frac{1}{2}} .
$$

If we suppose that the unspecified scalar multiplier of $\varphi$ has been chosen so that $\left(\varphi, u_{i}\right)$ is real, positive, and equal to $\left|\left(\varphi, u_{i}\right)\right|$, then

$$
|| \varphi-u_{i}||=\sqrt{ } 2\left[1-\left|\left(\varphi, u_{i}\right)\right|\right]^{\frac{1}{2}},
$$

so that our problem is equivalent to the determination of a positive lower bound to the magnitude of the projection of $\varphi$ on $u_{i}$.

\footnotetext{
*This work was supported in part by the Department of the Navy under contract Nord 7386 with the Bureau of Naval Weapons, and was conducted in part while the authors were consultants at the Institut Battelle, Geneva, Switzerland.

**Applied Physies Laboratory, Johns Hopkins University, Silver Spring, Md.

1 P.-O. Löwdin, Adv. Chem. Phys. II, 207 (1959).

${ }^{2}$ H. F. Weinberger, J. Research NBS 64B, 217 (1960).

3 T. Kato, J. Phys. Soc. Japan 4, 334 (1949).

4 N. Kryloff, Mém. des Sci. Math. 49 (1931).

5 E. Trefftz, Math. Ann. 108, 595 (1933).

${ }_{6}^{6}$ H. F. Weinberger, Inst. Fld. Dyn. and App. Math., Univ. of Md. Tech. Note BN-183 (1959).
} 
In order to obtain our estimates we require upper and lower bounds to a finite number of eigenvalues. We shall designate a lower bound to the $j$ th eigenvalue by $\lambda_{j}^{l}$ and an upper bound by $\lambda_{j}^{u}$; thus

$$
\lambda_{j}^{l} \leq \lambda_{j} \leq \lambda_{j}^{u}
$$

A condition sufficient to guarantee that $\lambda_{i}$ be nondegenerate is that $\lambda_{i-1}^{u}\left\langle\lambda_{i}^{l}\right.$ and $\lambda_{i+1}^{l}>\lambda_{i}^{u}$. Upper bounds are usually found by the Rayleigh-Ritz procedure, while lower bounds can often be obtained by a variety of procedures.

Let us choose $n \geq i$. By the spectral theorem we have ${ }^{7}$

$$
(A \varphi, \varphi)=\int_{-\infty}^{\infty} \lambda d\left(E_{\lambda} \varphi, \varphi\right) \geq \sum_{j=1}^{n} \lambda_{j}\left|\left(\varphi, u_{j}\right)\right|^{2}+\lambda_{n+1}\left[1-\sum_{j=1}^{n}\left|\left(\varphi, u_{j}\right)\right|^{2}\right] .
$$

Since the coefficients of $\lambda_{1}, \lambda_{2}, \ldots, \lambda_{n+1}$ are nonnegative, we may replace these eigenvalues by the lower bounds $\lambda_{1}^{l}, \lambda_{2}^{l}$, . . ., $\lambda_{n+1}^{l}$ to obtain

$$
(A \varphi, \varphi) \geq \sum_{j=1}^{n} \lambda_{j}^{l}\left|\left(\varphi, u_{j}\right)\right|^{2}+\lambda_{n+1}^{l}\left[1-\sum_{j=1}^{n}\left|\left(\varphi, u_{j}\right)\right|^{2}\right] .
$$

The inequality (3) may be solved for $\left|\left(\varphi, u_{i}\right)\right|^{2}$ in the form

$$
\left|\left(\varphi, u_{i}\right)\right|^{2} \geq \frac{\lambda_{n+1}^{l}-(A \varphi, \varphi)}{\lambda_{n+1}^{l}-\lambda_{i}^{l}}-\frac{1}{\lambda_{n+1}^{l}-\lambda_{i}^{l}} \sum_{j=1}^{n}\left(\lambda_{n+1}^{l}-\lambda_{j}^{l}\right)\left|\left(\varphi, u_{j}\right)\right|^{2},
$$

where $\Sigma^{\prime}$ means that the term for which $j$ equals $i$ has been omitted. This lower bound for $\left|\left(\varphi, u_{i}\right)\right|^{2}$ still involves unknown eigenvectors of $A$. This difficulty will now be overcome through use of the Schwarz inequality. For the arbitrary real number $\mu_{j}$ we have

$$
\left[\lambda_{j}-\mu_{j}\right]^{2}\left|\left(u_{j}, \varphi\right)\right|^{2}=\left|\left(\left[A-\mu_{j}\right] u_{j}, \varphi\right)\right|^{2}=\left|\left(u_{j},\left[A-\mu_{j}\right] \varphi\right)\right|^{2} \leq\left.||\left[A-\mu_{j}\right] \varphi\right|^{2},
$$

so that for $\mu_{j} \neq \lambda_{j}$ we have

$$
\left|\left(\varphi, u_{j}\right)\right|^{2} \leq \frac{\left\|\left[A-\mu_{j}\right] \varphi\right\|^{2}}{\left(\lambda_{j}-\mu_{j}\right)^{2}}
$$

We assume that $\varphi$ is not one of the eigenvectors $u_{1}, u_{2}, \ldots, u_{j-1}, u_{j+1}, \ldots, u_{n}$ and minimize the right hand side of (6) with respect to $\mu_{j}$. For the minimizing value given by

$$
\mu_{j}=\frac{(A \varphi, A \varphi)-\lambda_{j}(A \varphi, \varphi)}{(A \varphi, \varphi)-\lambda_{j}},
$$

the inequality (6) becomes

$$
\left|\left(\varphi, u_{j}\right)\right|^{2} \leq \frac{(A \varphi, A \varphi)-(A \varphi, \varphi)^{2}}{(A \varphi, A \varphi)-(A \varphi, \varphi)^{2}+\left[(A \varphi, \varphi)-\lambda_{j}\right]^{2}}
$$

Let us define numbers $\gamma_{j}$ by

so that

$$
\gamma_{j}=\left\{\begin{array}{cl}
\lambda_{j}^{l}-(A \varphi, \varphi), & (A \varphi, \varphi)<\lambda_{j}^{l} \\
0, & \lambda_{j}^{l} \leq(A \varphi, \varphi) \leq \lambda_{j}^{u} \\
(A \varphi, \varphi)-\lambda_{j}^{u}, & (A \varphi, \varphi)>\lambda_{j}^{u}
\end{array}\right\},
$$

Using these inequalities, the inequalities (8) can be replaced by the weaker ones

\footnotetext{
7 In the case that $A$ has only $n$ eigenvalues before the first limit point $\lambda_{*}$ of its spectrum, we replace $\lambda_{n+1}$ by $\lambda_{*}$ in (2) and the succeeding equations.
} 


$$
\left|\left(\varphi, u_{j}\right)\right|^{2} \leq \frac{(A \varphi, A \varphi)-(A \varphi, \varphi)^{2}}{(A \varphi, A \varphi)-(A \varphi, \varphi)^{2}+\gamma_{j}^{2}}, j=1,2, \ldots, n ;(j \neq i)
$$

Our lower bound for $\left|\left(\varphi, u_{i}\right)\right|^{2}$ is obtained by using the inequalities (10) in (4); we have ${ }^{8}$

$$
\left|\left(\varphi, u_{i}\right)\right|^{2} \geq \frac{\lambda_{n+1}^{l}-(A \varphi, \varphi)}{\lambda_{n+1}^{l}-\lambda_{i}^{l}}-\frac{(A \varphi, A \varphi)-(A \varphi, \varphi)^{2}}{\lambda_{n+1}^{l}-\lambda_{i}^{l}} \cdot \sum_{j=1}^{n}{ }^{\prime} \frac{\lambda_{n+1}^{l}-\lambda_{j}^{l}}{(A \varphi, A \varphi)-(A \varphi, \varphi)^{2}+\gamma_{j}^{2}} .
$$

When the right hand side of (11) is positive, it can be used to give an upper bound to $\left\|\varphi-u_{i}\right\|$.

\section{Some Special Estimates}

In those cases when there are an infinite number of eigenvalues converging to a finite limit point or diverging to infinity it is possible to make other estimates of $\left|\left(\varphi, u_{i}\right)\right|^{2}$ which contain infinite sums. When these sums converge and can be estimated numerically the resulting inequalities may be useful in applications. We briefly sketch two such results.

Let us consider the case when the initial spectrum of $A$ consists of an infinite number of eigenvalues $\lambda_{1} \leq \lambda_{2} \leq \ldots$ that converge to the finite limit point $\lambda_{*}$. From the spectral theorem we have

$$
(A \varphi, \varphi) \geq \sum_{d=1}^{\infty} \lambda_{j}^{l}\left|\left(\varphi, u_{j}\right)\right|^{2}+\lambda_{*}^{l}\left[1-\sum_{j=1}^{\infty}\left|\left(\varphi, u_{j}\right)\right|^{2}\right]
$$

where $\lambda_{j}^{l}$ and $\lambda_{*}^{l}$ are lower bounds to $\lambda_{j}$ and $\lambda_{*}$ respectively. If $\lambda_{i}$ is a simple eigenvalue and if $\lambda_{*}^{l}>\lambda_{i}^{l}$, then (10) and (12) give

$$
\left|\left(\varphi, u_{i}\right)\right|^{2} \geq \frac{\lambda_{*}^{l}-(A \varphi, \varphi)}{\lambda_{*}^{l}-\lambda_{i}^{l}}-\frac{(A \varphi, A \varphi)-(A \varphi, \varphi)^{2}}{\lambda_{*}^{l}-\lambda_{i}^{l}} \sum_{j=1}^{\infty} \frac{\lambda_{*}^{l}-\lambda_{j}^{l}}{(A \varphi, A \varphi)-(A \varphi, \varphi)^{2}+\gamma_{j}^{2}} .
$$

Since the $\gamma_{j}$ 's are again given by (9), the inequality (13) requires the knowledge of upper bounds to each $\lambda_{j}$ for which $(A \varphi, \varphi) \geq \lambda_{j}^{l}$.

When $A$ has a pure point spectrum diverging to infinity various estimates can be given. One of them starts from the Parseval equation

and leads directly to the estimate

$$
\left|\left(\varphi, u_{i}\right)\right|^{2}=1-\sum_{j=1}^{\infty}\left|\left(\varphi, u_{j}\right)\right|^{2}
$$

$$
\left|\left(\varphi, u_{i}\right)\right|^{2} \geq 1-\left[(A \varphi, A \varphi)-(A \varphi, \varphi)^{2}\right] \cdot \sum_{j=1}^{\infty}{ }^{\prime} \frac{1}{(A \varphi, A \varphi)-(A \varphi, \varphi)^{2}+\gamma_{j}^{2}} \cdot
$$

\section{Degenerate Eigenvalues}

We now suppose that $\lambda_{i}$ is either an eigenvalue of multiplicity $m$, or one of $m$ eigenvalues which lie close together. Let $p$ and $q$ be the largest nonnegative integers such that

$$
\lambda_{i}^{l} \leq \lambda_{i-p}^{u}
$$

and

$$
\lambda_{i}^{u} \geq \lambda_{i+q}^{l}
$$

8 The inequality (11) has the following immediate generalization. If $f(\lambda)$ is a real valued function which is monotonically increasing and $f(A)$ is the corresponding self-adjoint transformation, then

$$
\left|\left(\varphi, u_{i}\right)\right|^{2} \geq \frac{f\left(\lambda_{n+1}^{l}\right)-(f(A) \varphi, \varphi)}{f\left(\lambda_{n+1}^{l}\right)-f\left(\lambda_{i}^{l}\right)}-\frac{(A \varphi, A \varphi)-(A \varphi, \varphi)^{2}}{f\left(\lambda_{n+1}^{l}\right)-f\left(\lambda_{i}^{l}\right)} \sum_{j=1}^{n} \frac{f\left(\lambda_{n+1}^{l}\right)-f\left(\lambda_{j}^{l}\right)}{(A \varphi, A \varphi)-(A \varphi, \varphi)^{2}+\gamma_{i}^{2}} \cdot
$$


then $m=p+q+1$. One appropriate question that may be asked is "How closely is some eigenvector in the span of $u_{i-p}, u_{i-p+1}, \ldots, u_{i+q}$ approximated by a unit vector $\varphi$ ?" This is equivalent to finding a lower bound to the projection of $\varphi$ on the span of $u_{i-p}, \ldots, u_{i+q}$. It follows in the same way as (11) that

$$
\sum_{j=i-p}^{i+q}\left|\left(\varphi, u_{j}\right)\right|^{2} \geq \frac{\lambda_{n+1}^{l}-(A \varphi, \varphi)}{\lambda_{n+1}^{l}-\lambda_{i-p}^{l}}-\frac{(A \varphi, A \varphi)-(A \varphi, \varphi)^{2}}{\lambda_{n+1}^{l}-\lambda_{i-p}^{l}} \sum_{j=1}^{n} \frac{\lambda_{n+1}^{l}-\lambda_{j}^{l}}{(A \varphi, A \varphi)-(A \varphi, \varphi)^{2}+\gamma_{j}^{2}},
$$

where $n \geq i+q$ and $\Sigma^{\prime}$ means that the terms for which $j=i-p, i-p+1, \ldots, i+q$ have been omitted. The estimates (13) and (14) can be extended in the same way.

(Paper 66B1-67) 